\title{
EphB Receptors and Ephrin-B3 Regulate Axon Guidance at the Ventral Midline of the Embryonic Mouse Spinal Cord
}

\author{
Stephanie R. Kadison, ${ }^{1}$ Taija Mäkinen, ${ }^{3}$ Rüdiger Klein, ${ }^{3}$ Mark Henkemeyer, ${ }^{4}$ and Zaven Kaprielian ${ }^{1,2}$ \\ Departments of ${ }^{1}$ Neuroscience and ${ }^{2}$ Pathology, Albert Einstein College of Medicine, Bronx, New York 10461, ${ }^{3}$ Department of Molecular Neurobiology, Max \\ Planck Institute of Neurobiology, 82152 Martinsried, Germany, ${ }^{4}$ Center for Developmental Biology and Kent Waldrep Foundation Center for Basic Research \\ on Nerve Growth and Regeneration, University of Texas Southwestern Medical Center, Dallas, Texas 33136
}

EphB receptors and their ephrin-B ligands are required for midline guidance decisions at several rostrocaudal levels of the developing CNS. In the embryonic vertebrate spinal cord, ephrin-B3 is localized to the floor plate (FP) at the ventral midline (VM), ephrin-B1 and ephrin-B2 are expressed in the dorsal spinal cord, and decussated EphB receptor-bearing commissural axons navigate between these ventral and dorsal ephrin-B domains. Despite these compelling expression patterns, the in vivo role(s) for EphB and ephrin-B proteins in regulating the guidance of spinal commissural axons has not been established. Here, we use DiI $\left(1,1^{\prime}\right.$-dioctadecyl-3,3,3',3'tetramethylindocarbocyanine perchlorate) labeling to assess the pathfinding of commissural axons in the spinal cords of ephrin- $\mathrm{B}$ and EphB mutant mouse embryos. In mice lacking ephrin-B3 or multiple EphB receptors, a significant number of axons followed aberrant trajectories in the immediate vicinity of the VM. Furthermore, forked transverse commissural (FTC) axons, a unique class of commissural axons that continues to project in the transverse plane on the contralateral side of the FP, were present at a markedly higher frequency in ephrin-B3 and EphB mutants, compared with wild-type embryos. Neither the midline guidance errors nor excessive numbers of FTC axons were observed in the spinal cords of ephrin-B $3^{\text {lacz }}$ mice that express a truncated form of ephrin-B3, which is capable of forward but not reverse signaling. In contrast to the midline guidance defects observed in EphB and ephrin-B3 mutant embryos, wild-type-like contralateral projections were observed in mice lacking ephrin-B1 and/or ephrin-B2.

Key words: EphB; ephrin-B; mouse embryo; spinal cord; ventral midline; floor plate; commissural axon

\section{Introduction}

Contact-dependent interactions between Eph receptor tyrosine kinases and their membrane-bound ephrin ligands mediate a variety of signaling events in the developing CNS (Palmer and Klein, 2003). Transmembrane B-class ephrins preferentially bind EphB receptors, whereas A-class ephrins, which are tethered to the cell surface by a glycosylphosphatidylinositol linkage, preferentially bind EphA receptors (Flanagan and Vanderhaeghen, 1998; Himanen et al., 2004). In addition to classical forward signaling through the Eph receptor-bearing cell, Eph/ephrin binding can also transmit a reverse signal into the ephrin-expressing cell (Cowan and Henkemeyer, 2002; Davy and Soriano, 2005).

Several studies have implicated roles for Ephs and ephrins in

\footnotetext{
Received April 11, 2006; revised July 21, 2006; accepted July 25, 2006.

This work was supported by National Institutes of Health Grants R01 MH66332 and R01 EY017434 (M.H.) and R01 NS38505 (Z.K.), as well as by New Jersey Commission on Spinal Cord Research Grant 05-3042-SCR-E-0 (Z.K.). We thank Kosta Dobrenis and Joe Zavilowitz for confocal assistance, Cuiling Wang for assistance with statistical analyses, Annika Porthin for genotyping/breeding mice, and C. Mason (Columbia University, New York, NY) for critically reading this manuscript. We are also grateful to Renping Zhou (Rutgers University, New Brunswick, NJ) for providing ephrin-A mutant embryos.

Correspondence should be addressed to Stephanie R. Kadison, Department of Neuroscience, Albert Einstein College of Medicine, Kennedy Center, Room 616, 1410 Pelham Parkway, Bronx, NY 10461. E-mail: skadison@ umich.edu.

S. Kadison's present address: Cell and Developmental Biology, University of Michigan, 109 Zina Pitcher Place, Room 3678, Ann Arbor, MI, 48109. E-mail: skadison@umich.edu.

DOI:10.1523/JNEUROSCI.1569-06.2006

Copyright $\odot 2006$ Society for Neuroscience $\quad$ 0270-6474/06/268909-06\$15.00/0
}

axon guidance and commissure formation at the midline of the vertebrate CNS. In the developing Xenopus (Nakagawa et al., 2000) and mouse (Williams et al., 2003) visual systems, ephrin-B2 functions as a repulsive ligand at the optic chiasm midline that establishes the ipsilateral projection. Similarly, ephrin-B3 acts as a midline repellent in postnatal mice; disruption of ephrin-B3-mediated forward signaling promotes inappropriate midline recrossing of the corticospinal tract (Yokoyama et al., 2001) and the aberrant decussation of ipsilateral axons in the spinal cord proper (Kullander et al., 2003). Mice lacking EphA4, which can bind ephrin-B2/B3 and ephrin-As, phenocopy the corticospinal tract defects in ephrin-B3 knockouts (KOs) (Kullander et al., 2001). In addition, ephrin-Binduced reverse signaling is required for anterior commissure formation (Henkemeyer et al., 1996; Cowan et al., 2004), and both forward and reverse signaling elicted by EphB/ephrin-B interactions regulate corpus callosum development (Mendes et al., 2006).

In the developing mouse spinal cord, commissural axons follow a circumferential trajectory toward and across the floor plate (FP), a ventral midline (VM)-associated intermediate target, which they never recross. Subsequently, many commissural axons turn into the longitudinal plane and then project away from the FP along a diagonal path into the dorsal spinal cord, where they execute a second longitudinal turn (Kadison and Kaprielian, 2004; Nissen et al., 2005). We have shown that ephrin-B3 is lo- 
calized to the FP, ephrin-B1 and ephrin-B2 are expressed in the dorsal spinal cord, and EphB proteins are present on segments of commissural axons (Imondi et al., 2000; Imondi and Kaprielian, 2001; Jevince et al., 2006). We also demonstrated that ephrin-B proteins promote the collapse of commissural growth cones and that decussated commissural axons inappropriately invade dorsal regions of the spinal cord when EphB/ephrin-B interactions are perturbed in vitro. Together, these observations raised the possibility that ephrin-B3 functions as a repulsive barrier at the spinal cord midline and that ephrin-B proteins may constrain the orientation of commissural axons on the contralateral side of the FP.

Here, we use 1,1'-dioctadecyl-3,3,3',3'-tetramethylindocarbocyanine perchlorate (DiI) labeling to examine the pathfinding of commissural axons in the spinal cords of mice lacking B-class ephrins or EphB receptors. These analyses reveal that ephrin-B-induced forward signaling is required for midline guidance decisions but that EphB/ephrin-B interactions do not appear to be necessary for the proper elaboration of contralateral commissural projections.

\section{Materials and Methods \\ Mice}

EphB (Henkemeyer et al., 1996; Orioli et al., 1996; Williams et al., 2003) and ephrin-B3 (Yokoyama et al., 2001) mutant mice were generated/ genotyped as described previously. Ephrin- $B 1^{K O}$ mice were generated using conditional ephrin-B1 mice crossed with PGK-Cre mice and genotyped as described previously (Compagni et al., 2003). Because ephrinB2-deficient embryos die in utero around embryonic day 10 (E10) as a result of abnormal cardiovascular development (Adams et al., 1999), we used a conditional ephrin-B2 allele (Grunwald et al., 2003) and Nestin promoter-driven Cre recombinase to remove ephrin-B2 in the nervous system before E11.5 (Kramer et al., 2006). Ephrin-B1/2-NesCre doubles were generated by mating ephrin-B1 $1^{l x / l x} ; e$ phrin-B2 $2^{l x / l x}$ females to Nestin$C r e^{+} ;$ephrin- $B 1^{+/ Y} ;$ ephrin-B2 $2^{l x / l x}$ males. Because ephrin-B1 is X linked, Cre-mediated deletion of the ephrin-B1 allele in the progeny gives rise to hemizygous males (ephrinB $1^{l x / Y}$ ) and heterozygous females $\left(\right.$ ephrinB $\left.1^{l x /+}\right)$; therefore, only $\mathrm{Cre}^{+}$male embryos derived from these breedings were considered double mutants. Pregnant dams were killed by exposure to compressed carbon dioxide. Embryos were removed by cesarean section and immersed in PBS (in mM: $150 \mathrm{Na}_{2} \mathrm{HPO}_{4}, 20$ $\mathrm{NaH}_{2} \mathrm{PO}_{4}$, and $150 \mathrm{NaCl}, \mathrm{pH}$ 7.4). Open-book preparations were generated as described previously (Kadison and Kaprielian, 2004).

\section{Biochemistry}

Spinal cords derived from E12-E13 mice were homogenized by micropestle (Eppendorf, Westbury, NY) and solubilized in $50 \mathrm{~mm}$ Tris, $\mathrm{pH}$ 7.5, $120 \mathrm{~mm} \mathrm{NaCl}, 10 \%$ glycerol, and 1\% Triton X-100 supplemented with $10 \mathrm{~mm} \mathrm{NaF}, 1 \mathrm{~mm}$ vanadate, and Complete protease inhibitors (Roche Applied Science, Indianapolis, IN). Lysates were clarified by centrifugation and subjected to immunoprecipitation and/or SDS-PAGE (nonreducing) and Western blot analysis. Antibodies used in these experiments included goat anti-mouse ephrin-B1 or ephrin-B2 (R \& D Systems, Minneapolis, MN), rabbit anti-mouse ephrinB (Bruckner et al., 1997), and mouse anti- $\alpha$-tubulin (Sigma, St. Louis, MO). The immunocomplexes were detected using HRP-conjugated secondary antibodies (Jackson ImmunoResearch, West Grove, PA) and ECL chemistry (Amersham Biosciences, Arlington Heights, IL).

\section{Axon tracing}

Crystal and focal application of DiI (Invitrogen, San Diego, CA) were performed in open-book preparations at a position located either halfway between the FP and roof plate or two-thirds of the way from the FP to the roof plate as described previously (Kadison and Kaprielian, 2004).

\section{Microscopy and imaging}

DiI labeling was visualized through epifluorescence optics (Nikon Eclipse TE300; Nikon, Tokyo, Japan) using a Cy3/DiI optical filter
Table 1. Quantification of midline errors in EphB/ephrinB mutant mice

\begin{tabular}{|c|c|c|c|}
\hline Genotype & Number of embryos & Total cohorts & $\begin{array}{l}\% \text { Cohorts displaying } \\
\text { midline errors }\end{array}$ \\
\hline Wild type & 26 & 94 & $1(1 \%)$ \\
\hline ephrinB3 $3^{+/-}$ & 28 & 115 & $10(9 \%)^{*}$ \\
\hline ephrinB3 ${ }^{-1-}$ & 32 & 128 & $13(10 \%)^{*}$ \\
\hline ephrinB3 + /lacz & 12 & 43 & $0(0 \%)$ \\
\hline ephrinB3 ${ }^{\text {lacz }}$ & 10 & 34 & $1(3 \%)$ \\
\hline $\mathrm{EphB2}^{+/-} \mathrm{B3}^{-1-}$ & 16 & 66 & $5(8 \%)^{*}$ \\
\hline$E p h B 2^{-1-} B 3^{-1-}$ & 19 & 76 & $4(5 \%)$ \\
\hline $\mathrm{EphB1}^{-1-} \mathrm{B} 2^{+/-} \mathrm{B} 3^{-1-}$ & 20 & 78 & $8(10 \%)^{*}$ \\
\hline $\mathrm{EphB1}^{-1-} \mathrm{B} 2^{-1-} \mathrm{B3}^{-1-}$ & 17 & 68 & $10(15 \%)^{*}$ \\
\hline
\end{tabular}

${ }^{*} p<0.05 ; \chi^{2}$ approximation.

${ }^{a}$ Midline errors were defined as aberrant axon projections that were observed either while crossing the floor plate or within the vicinity of the midline (see Results and Materials and Methods for details).

(Chroma Technology Corporation, Brattleboro, VT). Black-and-white or color images were captured and processed as described previously (Kadison and Kaprielian, 2004). Confocal images of select DiI-labeled open-book preparations were acquired on a Fluoview 500 microscope (Olympus, Tokyo, Japan) using a Cy3 optical filter.

\section{Data analysis}

Midline/contralateral errors. A $\chi^{2}$ approximation was performed to determine the statistical significance of the relationship between the incidences of midline/contralateral errors in mutant embryos and wild-type (WT) embryos; $p<0.05$ was taken to indicate significance (Table 1 ). In general, a cohort (defined as the group of labeled axons resulting from a single application of DiI) was scored as exhibiting midline or contralateral errors when it contained at least one axon that did not travel along an intermediate longitudinal commissural (ILC)-, medial longitudinal commissural (MLC)-, bifurcating longitudinal commissural (BLC)-, or forked transverse commissural (FTC)-like trajectory (see Fig. 1, Schematic). More specifically, "midline errors" included axons that failed to accurately project orthogonally through the margins of the FP (see Fig. $1 D, F, G)$ or axons that elaborated aberrant FTC projections at the vicinity of the VM (see Fig. 1E). "Contralateral errors" included decussated axons that did not travel along WT contralateral commissural trajectories (see Fig. 1, Schematic, $A-C$ ).

Frequency of FTC projections. The percentage of embryos displaying FTC axons (see Fig. 2) was calculated by dividing the total number of embryos displaying at least one FTC axon by the total number of embryos for a given genotype. Because of the sample size, a Fisher's exact test was performed to determine the statistical significance of the relationship between the incidences of FTC projections observed in mutant and WT embryos; $p<0.05$ or $p<0.001$ was taken to indicate significance (see Fig. $2 E)$.

Shape of ILC projections. The distance at which ILC axons executed their second turn into the longitudinal plane was measured from the contralateral margin of the FP by drawing a ruler containing $50 \mu \mathrm{m}$ increments in Photoshop 7.0 (Adobe Systems, San Jose, CA) (see Fig. 3A) for each cohort. The average distance for a given genotype was then calculated (see Fig. $3 F$ ), and the mixed-effects model was used to determine whether these values varied significantly between genotypes; $p<$ 0.05 was taken to indicate significance. In all experiments involving mutant mice, at least four embryos of a given genotype were analyzed unless otherwise noted.

\section{Results}

Ephrin-B3 and EphB receptors are required for accurate pathfinding at the VM of the embryonic mouse spinal cord

In WT animals, spinal commissural axons elaborate at least four different contralateral projections in the embryonic mouse spinal cord (Fig. 1, Schematic). (1) Decussated ILC axons travel along an arcuate trajectory away from the FP and into the dorsal spinal cord, where they execute a second turn into the longitudinal plane. (2) After crossing the VM, MLC axons execute a single 

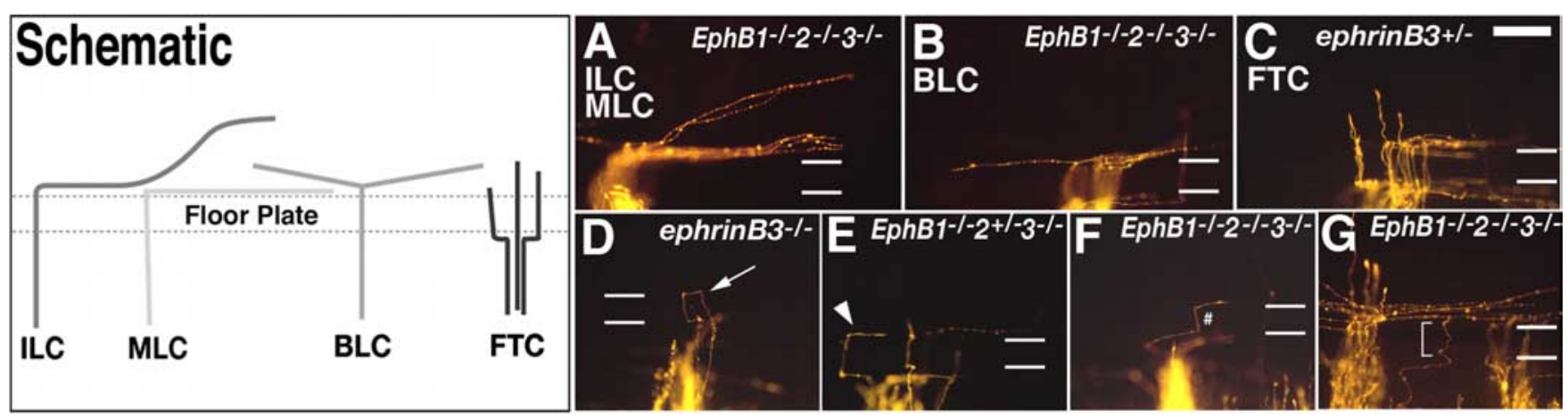

Figure 1. Commissural axons display a variety of pathfinding errors at the VM of the spinal cord in ephrin-B3 and EphB mutant mice. Schematic, Commissural axons elaborate four distinct commissural projections on the contralateral side of the FP in an open-book preparation derived from an E12.5-E13.5 WT mouse spinal cord: ILC, MLC, BLC, and FTC (Kadison and Kaprielian, 2004). $A-C$, Representative examples of WT-like contralateral projections observed in EphB and ephrin mutants. D-G, Representative examples of midline guidance errors (see Materials and Methods for details on scoring). Commissural axons were visualized through focal applications of Dil in open-book spinal cord preparations derived from E12.5-E13.5 ephrin-B3 and EphB mutant mice. The arrow in $\boldsymbol{D}$ points to an axon "looping" within the confines of the FP. The arrowhead in $\boldsymbol{E}$ identifies an apparently misguided FTC axon that has turned at the contralateral margin of the FP instead of continuing to grow in the transverse plane on the contralateral side of the VM. The \# sign in $\boldsymbol{F l a b e l s}$ an axon that is inappropriately zigzagging through the FP. The bracket in $\boldsymbol{G}$ delimits a particularly aberrant projection within the FP. Thin white lines demarcate the lateral edges of the FP. Scale bar, $100 \mu \mathrm{m}$.

right-angle turn and project for significant distances alongside the FP. (3) Decussated BLC axons split into rostrally and caudally directed projections. (4) FTC axons continue to project in the transverse plane after crossing the FP. When multiple FTC axons decussate, they form a forked-shaped projection (Fig. 1, Schematic) (Kadison and Kaprielian, 2004).

We have shown previously that ephrin-B3 mRNA and ephrin-B protein are localized to the FP and $\mathrm{EphB}$ receptors are preferentially expressed on decussated segments of commissural axons in the embryonic mouse spinal cord (Imondi et al., 2000; Imondi and Kaprielian, 2001; Jevince et al., 2006). These expression patterns raised the possibility that interactions between ephrin-B3 and EphB receptors regulate spinal commissural axon guidance in vivo. Accordingly, we performed axon tracing studies to determine whether spinal commissural axons appropriately cross the midline and elaborate WT-like contralateral projections in mice lacking ephrin-B3 or EphB receptors. Specifically, we unilaterally applied focal amounts of DiI to open-book preparations derived from E12.5-E13.5 mouse embryos representing various genotypes (Table 1). We then scored each cohort (group of axons labeled by an application of DiI) for midline and contralateral guidance errors (see Materials and Methods for details). There was no significant difference between the frequency of contralateral errors observed in the spinal cords of B-class Eph/ephrin mutant and WT embryos (data not shown). In contrast, commissural axon-containing cohorts in EphB2 $2^{+/-} 3^{-/-}$, EphB1 $1^{-/-} 2^{+/-} 3^{-/-}, E p h B 1^{-/-} 2^{-/-} 3^{-1-}$, ephrin-B3 $3^{-/-}$, and ephrin- $B 3^{+/-}$mouse embryos exhibited a significantly greater number of midline defects, as compared with their WT counterparts. Representative examples of these errors are illustrated in Figure 1. The misguided axons projected along aberrantly shaped FTC trajectories, zigzagged, or looped within the VM (Fig. $1 D-G)$. EphB triple KOs displayed the highest percentage (15\%) (Table 1) of midline errors.

To determine whether the disruption of forward or reverse signaling accounts for the midline phenotype observed in ephrin$B 3^{-1-}$ mice, we assessed axon pathfinding in a mouse that selectively lacks the cytoplasmic domain of ephrin-B3 (ephrin-B3 ${ }^{\text {lacz }}$ ) and is thus incapable of reverse signaling (Yokoyama et al., 2001). The lack of significant numbers of midline errors in these particular animals indicates that the extracellular and transmembrane domain of ephrin-B3 is sufficient to "rescue" the midline pheno- type displayed by ephrin-B3 null mice (Table 1 ). Together, these results imply that ephrin-B3 acts as a ligand to stimulate EphB forward signaling during axon pathfinding at the VM of the embryonic mouse spinal cord.

FTC projections were more frequently observed in ephrin-B3 null and $E$ phB mutant mouse embryos compared with WT littermates

We reported previously that FTC axons represent a rare population of commissural axons in the WT embryonic mouse spinal cord (Kadison and Kaprielian, 2004). In the DiI labeling studies described above, we observed more labeled FTC axons in the spinal cords of EphB/ephrin-B mutant embryos than in WT littermates. For this particular analysis, we scored the number of embryos instead of the number of cohorts displaying FTC projections because our previous studies in WT mice indicated that this would be a more conservative estimate of FTC frequency. Our analyses revealed that a larger proportion of ephrin-B3 $3^{-1-}$ $E p h B 2^{+/-} 3^{-/-}, E p h B 1^{-/-} 2^{+/-} 3^{-/-}$, and EphB1 $1^{-/-} 2^{-/-} 3^{-/-}$ embryos displayed FTC projections compared with WT littermates (Fig. 2E). Representative examples of open-book preparations derived from embryos containing labeled FTC axons are shown in Figure 2. Notably, ephrin-B $3^{\text {lacz }}$ homozygous embryos did not exhibit an increased incidence of FTC axons, further supporting a role for ephrin-B3 functioning as a ligand to stimulate forward signaling in axon guidance at the VM of the spinal cord.

\section{ILC axons follow appropriate contralateral trajectories in ephrin-B mutant mice}

We previously showed that decussated segments of ILC axons execute their second turn into the longitudinal plane alongside a dorsal domain of ephrin-B protein visualized through the use of EphB affinity probes (Imondi et al., 2000; Imondi and Kaprielian, 2001). Complementary in situ hybridization and immunohistochemical analyses indicate that the dorsal ephrin-B domain is likely to reflect the distribution of ephrin-B1 and ephrin-B2, but not ephrin-B3 (Imondi et al., 2000; Jevince et al., 2006). These observations, together with the results of in vitro perturbation experiments, raised the possibility that ephrin-B1 and ephrin-B2 define a repulsive boundary that constrains the orientation of 


\begin{tabular}{|c|c|c|c|c|}
\hline wild-type & B ephrinB3- & Genotype & No. Embryos & $\begin{array}{c}\text { \% Embryos Displaying } \\
\text { FTC axons }\end{array}$ \\
\hline & & wild-type & 26 & " $10 \%$ \\
\hline & & ephrin B $^{\text {th }}$ & 28 & $36 \%$ \\
\hline & & ephrinB3 ${ }^{\prime \prime}$ & 32 & $53 \%{ }^{*}$ \\
\hline & 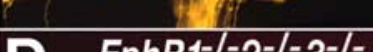 & ephrinB33 ${ }^{\text {thacz }}$ & 12 & $17 \%$ \\
\hline & D & ephrinB3 $3^{\text {lacz }}$ & 10 & $10 \%$ \\
\hline & & EphB2 $^{\prime \prime}$ B3 $^{\prime \prime}$ & 16 & $44 \%^{*}$ \\
\hline & & EphB2 $^{\prime}$ B3 $^{\prime}$ & 19 & $32 \%$ \\
\hline & & $\mathrm{EphB1}^{\prime-1} \mathrm{~B}^{+1-} \mathrm{B3}^{\prime}$ & 10 & $70 \%{ }^{\star}$ \\
\hline Wy & - & $E p h B 1^{\prime \prime} \mathrm{B}^{\prime}{ }^{\prime} \mathrm{B}^{\prime}$ & 14 & $86 \%{ }^{\star \star}$ \\
\hline
\end{tabular}

Figure 2. WT-like FTC axons are present in mice lacking ephrin-B3 or EphB receptors. $A-D$, FTC projections visualized through focal applications of Dil in open-book spinal cord preparations

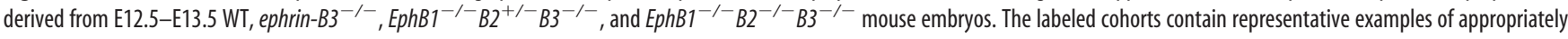
shaped FTC projections in both WT and mutant mice. Although these examples contain multiple FTC axons, an embryo was classified as containing an FTC projection if it displayed at least one transversely oriented axon. $\boldsymbol{E}$, FTC projections were more frequently observed in mice lacking ephrin-B3 or multiple EphB receptors. ${ }^{*} p<0.05$, ${ }^{* *} p<0.001$ (Fisher's exact test). Thin white lines demarcate the lateral edges of the FP. Scale bar, $100 \mu \mathrm{m}$.
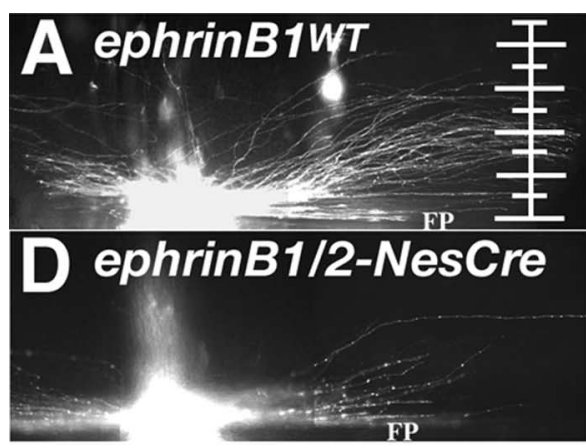
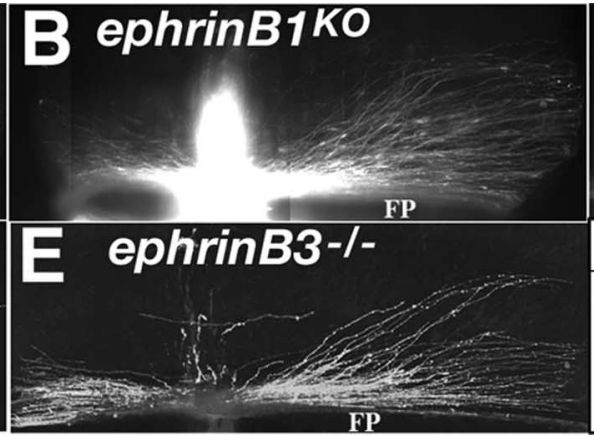

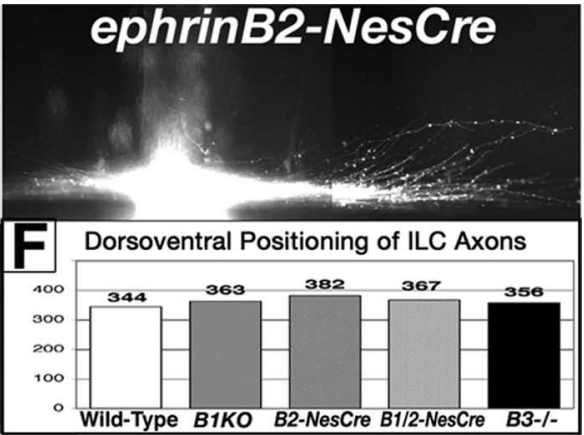

Figure 3. Spinal commissural axons elaborate WT-like ILC projections in B-class ephrin mutant mice. $\boldsymbol{A}-\boldsymbol{E}$, Open-book views of contralateral commissural projections visualized with crystal applications of Dil in spinal cords derived from E12.5-E13.5 ephrin-B conditional mutant and ephrin-B $7^{W T}$ (control) embryos. Similarly shaped contralateral commissural projections are present in each preparation. All micrographs represent single-plane images captured with a conventional fluorescent microscope, except $\boldsymbol{E}$. The image in $\boldsymbol{E}$ represents a $Z$-stack captured with a confocal microscope. The distance from the contralateral margin of the FP to the position at which ILC axons executed their second longitudinal turn was measured using the ruler depicted in $\boldsymbol{A}$. $\boldsymbol{F}$, There was no significant difference between these average distances in mutants and control embryos. FP indicates the location of the FP, and each increment on the ruler in $\boldsymbol{A}$ is $50 \mu \mathrm{m}$.

longitudinally projecting ILC axons (Imondi and Kaprielian, 2001).

To perform an in vivo test of this hypothesis, we unilaterally applied DiI crystals to open-book preparations derived from E12.5-E13.5 ephrin-B1 ${ }^{W T}$, ephrin-B1 ${ }^{K O}$, ephrin-B2-NesCre, ephrin-B1/B2-NesCre, and ephrin-B3 ${ }^{-1-}$ embryos. Importantly, a Western blot analysis revealed that both ephrin-B1 and ephrin-B2 (except for residual ephrin-B2, which is likely associated with blood vessels and therefore not targeted by the NesCre strategy) were essentially eliminated from the conditional mutants (supplemental Fig. 1, available at www. jneurosci.org as supplemental material). The crystal, as opposed to focal, application of DiI resulted in the labeling of large numbers of appropriately shaped ILC axons in openbook preparations derived from embryos of each genotype (Fig. 3). To quantitatively compare the labeled ILC projections in mutant and WT embryos, we measured the distance from the contralateral margin of the FP to the position at which these axons turned into the longitudinal plane in the dorsal spinal cord (Fig. 3A). This analysis revealed no significant difference between these average distances in mutant and WT embryos (Fig. $3 F)(p=0.93)$, suggesting that B-class ephrins are not likely to function as a repulsive barrier that constrains the orientation of ILC axons.

\section{Discussion}

In the developing vertebrate CNS, EphB receptors and ephrin-B ligands are required for the proper guidance of several classes of midline-crossing axons (see Introduction). Despite their compelling expression patterns (Imondi et al., 2000; Jevince et al., 2006), an in vivo role for $\mathrm{EphB}$ and ephrin-B proteins in the guidance of spinal commissural axons has not been established. We show here that B-class ephrins are not required to constrain the growth of decussated ILC axons. In contrast, however, in the spinal cords of mice lacking particular B-class Eph receptors or ephrin-B3, a significant number of axons follow aberrant trajectories in the immediate vicinity of the VM. This includes a substantial increase in FTC axons, which extend in the transverse plane after crossing the FP and represent a relatively rare commissural subtype in WT mice. Collectively, these midline-associated phenotypes are conspicuously absent in a mouse expressing a truncated form of ephrin-B3 (ephrin-B $3^{\text {lacz }}$ ) that is capable of forward but not reverse signaling. Together, these data suggest that, in the embryonic mouse spinal cord, B-class ephrins do not appear to have a significant role in shaping contralateral commissural projections, but ephrin-B3-mediated forward signaling is required for the proper guidance of axons at the VM. 


\section{B-class ephrins do not appear to regulate commissural axon pathfinding on the contralateral side of the FP}

On the contralateral side of the FP, EphB receptor-bearing ILC axons navigate between two ephrin-B domains: a stripe of ephrin-B3 at the FP and a broad region of ephrin-B1/2 expression in the dorsal spinal cord (Imondi and Kaprielian, 2001). Because ILC axons execute their second turn into the longitudinal plane alongside the dorsal ephrin-B domain, we proposed previously that ephrin-B proteins compose a repulsive boundary in the dorsal spinal cord that constrains the growth of longitudinally projecting ILC axons. The ability of B-class ephrins to collapse commissural growth cones (Imondi et al., 2000) and the observation that decussated commissural axons overshoot the dorsal ephrin-B boundary when EphB/ephrin-B interactions are perturbed in vitro (Imondi and Kaprielian, 2001) support this hypothesis. Inconsistent with this model, we show here that EphB/ephrin-B mutant embryos display WT-like contralateral commissural projections and that decussated ILC axons are properly positioned along the dorsoventral axis of the spinal cord. These findings raise the possibility that ephrin-As, which are also expressed in a dorsal domain of the embryonic mouse spinal cord (Yue et al., 1999), or other contact-dependent repulsive cues constrain the growth of decussated ILC axons. Notably, however, preliminary analyses reveal the presence of WT-like contralateral commissural projections in ephrin-A2/A5 double mutant embryos (data not shown).

\section{EphB receptors and ephrin-B3 regulate midline guidance decisions in the embryonic mouse spinal cord}

We previously used ligand and receptor affinity probes to demonstrate that EphB receptors and ephrin-B ligands are reciprocally expressed on postcrossing segments of commissural axons and FP cells (Imondi et al., 2000; Imondi and Kaprielian, 2001). Given that endogenous receptor-ligand interactions could potentially mask affinity probe binding sites, we recently used a battery of antibodies specific for EphB or ephrin-B proteins to verify these expression patterns. The results of these immunohistochemical analyses are generally consistent with EphB1, EphB2, and EphB3 being preferentially expressed on decussated segments of commissural axons (Jevince et al., 2006). However, in contrast to affinity probe binding, these analyses show that EphB1-3 are each expressed on the ventral commissure, which is composed of axons in the act of crossing the FP (Jevince et al., 2006). A plausible explanation for this apparent discrepancy is that $\mathrm{EphB}$ receptors residing on decussating commissural axons may engage FPassociated ephrin-B proteins, rendering them inaccessible to ligand affinity probes. Consistent with previous in situ hybridization studies (Imondi et al., 2000), antibody labeling also indicates that ephrin-B3 is likely to be the predominant ephrin-B expressed by FP cells.

We show here that mice lacking EphB receptors or ephrin-B3 exhibit a variety of midline guidance defects, including looping or zigzagging within the FP. This behavior is consistent with the absence of an inhibitory midline barrier, presumably ephrin-B3 (in the case of ephrin-B3 homozygotes), or the inability of commissural axons to respond to midline-associated ephrin-B3 (in EphB-lacking mice). The increased number of FTC axons in the mutant embryos would also be consistent with ephrin-B3 functioning as a midline repellent in WT embryos that normally exclude EphB receptor-expressing ipsilateral axons from the VM. Of direct relevance to these latter interpretations, ephrin-B ligands have been localized to the VM at several anteroposterior levels of the CNS, where they function as repulsive barriers for EphB receptor-bearing axons (Henkemeyer et al., 1996; Nakagawa et al., 2000; Kullander et al., 2001, 2003; Yokoyama et al., 2001; Williams et al., 2003; Cowan et al., 2004; Mendes et al., 2006). Furthermore, the loss of ephrin-B3 at the midline of the postnatal mouse spinal cord results in an increased number of decussated EphA4-expressing axons, which normally remain on the ipsilateral side of the FP in WT animals (Kullander et al., 2003).

\section{Ephin-B3-mediated forward signaling is required for axon guidance at the midline of the embryonic spinal cord}

It is now well established that B-class ephrins can function as ligands or receptors and thereby mediate forward or reverse signaling, respectively (Cowan and Henkemeyer, 2002). To determine whether the midline phenotypes observed in ephrin-B3 null embryos result from the disruption of forward or reverse signaling, we analyzed a mutant mouse that lacks the cytoplasmic domain of ephrin-B3, which disables ephrinB3-mediated reverse signaling (Yokoyama et al., 2001). Expression of truncated ephrin-B3 rescued the midline phenotype, suggesting that ephrin-B3-mediated forward but not reverse signaling is likely to be responsible for the observed $\mathrm{VM}$-associated defects. It is interesting to note in this regard that ephrin-B3-mediated forward signaling is also required to prevent the recrossing of corticospinal axons (Yokoyama et al., 2001) and the inappropriate decussation of ipsilateral axons in the spinal cord proper (Kullander et al., 2003).

\section{References}

Adams RH, Wilkinson GA, Weiss C, Diella F, Gale NW, Deutsch U, Risau W, Klein R (1999) Roles of ephrinB ligands and EphB receptors in cardiovascular development: demarcation of arterial/venous domains, vascular morphogenesis, and sprouting angiogenesis. Genes Dev 13:295-306.

Bruckner K, Pasquale EB, Klein R (1997) Tyrosine phosphorylation of transmembrane ligands for Eph receptors. Science 275:16401643.

Compagni A, Logan M, Klein R, Adams RH (2003) Control of skeletal patterning by ephrinB1-EphB interactions. Dev Cell 5:217-230.

Cowan CA, Henkemeyer M (2002) Ephrins in reverse, park, and drive. Trends Cell Biol 12:339-346.

Cowan CA, Yokoyama N, Saxena A, Chumley M, Silvany R, Baker L, Srivastava D, Henkemeyer M (2004) Ephrin-B2 reverse signaling is required for axon pathfinding and cardiac valve formation but not early vascular development. Dev Biol 27:263-271.

Davy A, Soriano P (2005) Ephrin signaling in vivo: look both ways. Dev Dyn 232:1-10.

Flanagan JG, Vanderhaeghen P (1998) The ephrins and Eph receptors in neural development. Annu Rev Neurosci 21:309-345.

Grunwald IC, Korte M, Adelmann G, Pueck A, Kullander K, Adams RH, Frotscher M, Bonhoeffer T, Klein R (2003) Hippocampal plasticity requires postsynaptic ephrinBs. Nat Neurosci 7:33-40.

Henkemeyer M, Orioli D, Henderson JT, Saxton TM, Roder J, Pawson T, Klein R (1996) Nuk controls pathfinding of commissural axons in the mammalian central nervous system. Cell 86:35-46.

Himanen JP, Chumley MJ, Lackmann M, Li C, Barton WA, Jeffrey PD, Vearing C, Geleick D, Feldheim DA, Boyd AW, Henkemeyer M, Nikolov DB (2004) Repelling class discrimination: ephrin-A5 binds to and activates EphB2 receptor signaling. Nat Neurosci 7:501-509.

Imondi R, Kaprielian Z (2001) Commissural axon pathfinding on the contralateral side of the floor plate: a role for B-class ephrins in specifying the dorsoventral position of longitudinally-projecting commissural axons. Development 128:4859-4871.

Imondi R, Wideman C, Kaprielian Z (2000) Complementary expression of transmembrane ephrins and their receptors in the mouse spinal cord: a 
possible role in constraining the orientation of longitudinally projecting axons. Development 127:1397-1410.

Jevince AR, Kadison SR, Pittman AJ, Chien C-B, Kaprielian Z (2006) Distribution of EphB Receptors and ephrin-B1 in the developing vertebrate spinal cord. J Comp Neurol 497:734-750.

Kadison SR, Kaprielian Z (2004) Diversity of contralateral commissural projections in the embryonic rodent spinal cord. J Comp Neurol 472:411-422.

Kramer ER, Knott L, Su F, Dessaud E, Krull CE, Helmbacher F, Klein R (2006) Cooperation between GDNF/Ret and ephrinA/EphA4 signals for motor-axon pathway selection in the limb. Neuron 50:35-47.

Kullander K, Croll SD, Zimmer M, Pan L, McClain J, Hughes V, Zabski S, DeChiara TM, Klein R, Yancopoulos GD, Gale NW (2001) Ephrin-B3 is the midline barrier that prevents corticospinal tract axons from recrossing, allowing for unilateral motor control. Genes Dev 15:877-888.

Kullander K, Butt SJ, Lebret JM, Lundfald L, Restrepo CE, Rydstrom A, Klein R, Kiehn O (2003) Role of EphA4 and EphrinB3 in local neuronal circuits that control walking. Science 299:1889-1892.

Mendes SW, Henkemeyer M, Liebl D (2006) Multiple Eph receptors and B-class ephrins regulate midline crossing of corpus callosum fibers in the developing mouse forebrain. J Neurosci 26:882-892.
Nakagawa S, Brennan C, Johnson KG, Shewan D, Harris WA, Holt CE (2000) Ephrin-B regulates the ipsilateral routing of retinal axons at the optic chiasm. Neuron 25:599-610.

Nissen UV, Mochida H, Glover JC (2005) Development of projectionspecific interneurons and projection neurons in the embryonic mouse and rat spinal cord. J Comp Neurol 483:30-47.

Orioli D, Henkemeyer M, Lemke G, Klein R, Pawson T (1996) Sek4 and Nuk receptors cooperate in guidance of commissural axons and in palate formation. EMBO J 15:6035-6049.

Palmer A, Klein R (2003) Multiple roles of ephrins in morphogenesis, neuronal networking, and brain function. Genes Dev 17:1429-1450.

Williams SE, Mann F, Erskine L, Sakurai T, Wei S, Rossi DJ, Gale NW, Holt CE, Mason CA, Henkemeyer M (2003) Ephrin-B2 and EphB1 mediate retinal axon divergence at the optic chiasm. Neuron 39:919-935.

Yokoyama N, Romero MI, Cowan CA, Galvan P, Helmbacher F, Charnay P, Parada LF, Henkemeyer M (2001) Forward signaling mediated by ephrin-B3 prevents contralateral corticospinal axons from recrossing the spinal cord midline. Neuron 29:85-97.

Yue Y, Su J, Cerretti DP, Fox GM, Jing S, Zhou R (1999) Selective inhibition of spinal cord neurite outgrowth and cell survival by the Eph family ligand ephrin-A5. J Neurosci 19:10026-10035. 